Strafvollzug offen. So existiert ein rechtsstaatlicher oder - vorsichtiger ausgedrückt ein rechtlich geordneter und überschaubarer Bereich immer nur unter dem Vorbehalt des Politischen. ${ }^{334}$

Vor diesem Hintergrund vertrat der Verfasser die Ansicht, dass die Beurteilung des Politischen sich jedweder "rechtlichen Normierbarkeit" entzog und so aus diesem Grund folgerichtig »Sondergerichte« entstanden, die sich an »kein positives Recht gebunden« fühlten und zu »reinen Verkörperungen« der sogenannten Führergerichtsbarkeit wurden. Die spolitische< Polizei und Gerichte griffen immer dann ein, wenn das "gesetzte Recht « versagte, es also zu einem Vorgehen oder zu einer Verurteilung entsprechend der politischen Notwendigkeit nicht ausreichte. Für Hofer hatte die NSJustiz einen »doppelten Boden«: Unzählige Menschen hatten es am eigenen Leibe erfahren, als sie nach einer »Verurteilung oder gar nach einer Freisprechung« durch ein »ordentliches Gericht« an der »Pforte des Gerichtsgebäudes von der Gestapo in Empfang genommen wurden« (S. 58). Das »Phänomen der >doppelten Rechtsordnung« war natürlich besonders deutlich »in der Behandlung des jüdischen Bevölkerungsteiles « $\mathrm{zu}$ sehen. Hierbei trat im Hinblick auf das Parteiprogramm der NSDAP, das "für Juden Fremdenrecht gefordert hatte«, eine Verschärfung ein. Nämlich ab 1938, als die Juden in Deutschland »im Zustand faktischer Rechtlosigkeit zu leben hatten«. Und in diesem Kontext schrieb er zum Abschluss seines Aufsatzes: "Eine ähnliche, doppelte Rechtsordnung schwebte Hitler im Verhältnis des deutschen Volkes zu den zu unterwerfenden Ostvölkern vor, worüber man Einzelheiten besonders in den >Tischgesprächen, aber auch in zahlreichen Verordnungen und Verfügungen, die während des Krieges erlassen wurden, nachlesen kann. ${ }^{335}$

\title{
1.10 Kommentierende Zusammenfassung
}

Im Folgenden geht es darum, das in den vorangehenden Kapiteln präsentierte empirische Material des Monat zur Geschichte des >Dritten Reiches kommentierend zusammenzufassen. Nicht zuletzt aus Raumgründen kann hierbei allerdings nicht auf jeden einzelnen Beitrag eingegangen werden. Aus demselben Grund wird bei der kommentierenden Zusammenfassung, die einem chronologischen Prinzip folgt, d.h., die jeweiligen Abschnitte werden nacheinander abgehandelt, in erster Linie nur auf zentrale historische Aspekte und Aussagen sowie Einschätzungen der Autoren aus den Veröffentlichungen eingegangen. Hierbei müssen also Schwerpunkte gesetzt werden. Zum Teil werden aus einzelnen Beiträgen nur die wesentlichen Feststellungen sowie Deutungen wiedergegeben und kommentiert, die grundsätzliche Erkenntnisse und Deutungen im Hinblick auf die »Analyse des Nationalsozialismus « im Monat ermöglichen, die wiederum - vor dem Hintergrund der zeitgenössischen wissenschaftlichen Forschungserkenntnisse zur Geschichte des `Dritten Reiches` - die Grundlage bilden für das abschließende in Thesen vorgetragene »analytische Resümee«.

Einer ersten generellen Einschätzung zufolge kann hinsichtlich der Analyse des Nationalsozialismus im Monat festgestellt werden, dass in den meisten Veröffentlichungen zu diesem Thema ein genuin personenzentrierter Blick eingenommen wurde. 
Gemeinhin wurde nämlich in allererster Linie die unmittelbare Führungs- und Herrschaftselite des >Dritten Reiches`, also Hitler, Göring, Himmler oder auch Goebbels, in den Fokus gerückt. Hier wiederum spielte freilich Hitler die alles überragende Rolle, was sich auch in den immer wieder genannten Begriffen und Kategorien wie »HitlerStaat «, »Führerstaat«, »Hitler-Herrschaft« oder »Hitlerismus« zur Charakterisierung des NS-Herrschaftssystems niederschlug. Das heißt, bei der Beschreibung und Analyse des NS-Herrschaftssystems der Jahre 1933 bis 1945 stand nach Auffassung des Monat bzw. der meisten Autoren der Zeitschrift im Grunde genommen, neben dem »anonymen« SS-Apparat unter der Führung Himmlers, ausschließlich Hitler im Mittelpunkt. Nach dem Motto, dass einzelne »Männer Geschichte machen«, traf nach Meinung der meisten Autoren ausschließlich Hitler alle wichtigen und unwichtigen Entscheidungen in den Jahren 1933 bis 1945, sodass beispielsweise die nationalsozialistische Machteroberung im Januar 1933 und der Zweite Weltkrieg sowie die insbesondere nach dem Überfall auf die Sowjetunion im Juni 1941 begangenen Massenverbrechen ausschließlich als das Werk Hitlers betrachtet wurden. Kurzum: Der Nationalsozialismus war in den Augen des Monat im Grunde genommen ein »Ein-Mann-Unternehmen«.

In nicht seltenen Fällen wurde bei der Thematisierung, Beschreibung und Analyse des Nationalsozialismus eine totalitarismustheoretische Perspektive eingenommen. Vor der Folie der Entwicklung der sowjetischen Geschichte nach der Russischen Revolution von 1917 wurde zum Beispiel auch von den beiden NS-Experten des Monat, Trevor-Roper und Hofer, immer wieder der Vergleich mit dem sowjetischen Herrschaftsregime unter Lenin und Stalin angestellt; exemplarisch war in diesem Zusammenhang auch die Rezension der Tagebücher von Goebbels von Richard H. S. Crossmann in der ersten Ausgabe des Monat und besaß insofern einen programmatischen Charakter.

Bei der Frage, welche thematischen Schwerpunkte bei der historischen Untersuchung und Beleuchtung der Genesis des Nationalsozialismus gesetzt wurden und über welche Aspekte, Bereiche, Abschnitte und Ereignisse generell geschrieben wurde, kann auf der Grundlage des empirischen Materials des Monat grundsätzlich festgestellt werden: Die Vorgeschichte, die zur Ernennung Hitlers zum Reichskanzler am 30. Januar 1933 führte, wurde durchaus angesprochen. Indes: Mit den genauen historischpolitischen und -gesellschaftlichen Voraussetzungen, die zur Auflösung der Weimarer Republik führten, setzte man sich nicht grundsätzlich auseinander. Im Großen und Ganzen wurden hierbei die Folgen des Versailler Vertrags in den Mittelpunkt gestellt. Mit anderen Worten: Wenn die komplexen historischen Voraussetzungen für das Aufkommen der nationalsozialistischen Massenbewegung zur Sprache kamen und der Versuch gemacht wurde, zu erklären, warum »Hitler möglich wurde« und zum Reichskanzler aufsteigen konnte, war zumeist von den entscheidenden Konsequenzen der aus dem Versailler Vertrag resultierenden Verpflichtungen für Deutschland die Rede. Nur ein Beitrag beschrieb und analysierte explizit das Ende der Weimarer Republik: Ferdinand Friedensburg in seiner Veröffentlichung Woran scheiterte die Republik von Weimar? Darüber hinaus wurden in diesem Zusammenhang im Monat die historischen Bedingungen, die die nationalsozialistische »Machtergreifung«möglich machten, im Grunde genommen nur indirekt angesprochen; wie zum Beispiel in dem Beitrag Der Führer persönlich. Gedanken beim Lesen zweier Biographien von Lüthy, in dem er unter anderem den Aufstieg Hitlers in der NSDAP sowie den der nationalsozialistischen Massenbewegung in den 1920er-Jahren vor dem Hintergrund der republikfeindlichen politischen Verhältnisse Münchens beleuchtete. 
Die politische Entwicklung des Nationalsozialismus nach Hitlers Ernennung zum Reichskanzler am 30. Januar 1933 untersuchte oder thematisierte Der Monat fast ausschließlich zum einen im Kontext des Reichstagsbrandes im Februar 1933 und zum anderen anhand der »Röhm-Affäre« im Juni 1934. Beide Ereignisse gehörten zur Phase der eigentlichen Machtkonsolidierung des Nationalsozialismus. Die ausdrücklichen Hinweise von Hofer in Der »völkische Rechtsstaat« zur innenpolitischen Entwicklung des »Führerstaates" sowie zur NS-Terrorpolitik stellten eine Ausnahme dar. Neben einzelnen "nebenbei« gemachten Hinweisen über Gewalt- bzw. Verfolgungsmaßnahmen gegen Kommunisten, Sozialdemokraten und gegen Juden in Deutschland sowie über nationalsozialistische Konzentrationslager in Veröffentlichungen, die nicht ausdrücklich die Geschichte des Nationalsozialismus zum Gegenstand hatten, besaß sowohl die konkrete innenpolitische als auch außenpolitische Entwicklung Deutschlands unter Hitler in den Jahren 1933 bis 1938 im Monat keine Bedeutung. In diesem Zusammenhang stellte die Veröffentlichung von Rohan Butler zum Fall Fritzsch im Jahre 1938 eine weitere Ausnahme dar.

Ganz im Gegensatz hierzu erlangte insbesondere die tatsächliche außenpolitische Entwicklung Deutschlands in den Jahren 1938 bis 1945 eine - man könnte sagen überragende Bedeutung. Denn zur politischen und diplomatischen Vorgeschichte sowie zum Zweiten Weltkrieg selbst, einschließlich der Memoirenliteratur und des militärisch-konservativen Widerstandes vom »20. Juli«, veröffentlichte die Zeitschrift Der Monat die allermeisten Artikel. Dieser Themenkomplex wurde abgesehen von der Vorabveröffentlichung der Studie Diplomatie aufschiefer Bahn von Joachim G. Leithäuser in der Regel in Form einer Buchrezension bzw. eines Rezensionsaufsatzes behandelt.

Wie gezeigt werden konnte, wurden im Kontext der Auseinandersetzung mit dem Zweiten Weltkrieg auch die insbesondere nach dem Überfall auf die Sowjetunion in Gang gekommenen Massenverbrechen an der osteuropäischen (Zivil-)Bevölkerung durchaus angesprochen. Ebenfalls beleuchtet wurde zudem das Kernereignis des Nationalsozialismus, die Vernichtung der europäischen Juden. Die Ermordung von sechs Millionen Juden wurde nicht nur von einigen jüdischen Autoren (wie zum Beispiel von Arendt, Aron oder Laqueur), sondern auch von nichtjüdischen Autoren (exemplarisch sei hier Lüthy genannt) festgestellt. Hierbei insistierten einige Autoren (unter anderem Alfred Kazin) auf den einzigartigen Charakter der Ermordung der europäischen Juden und hoben die nationalsozialistischen Konzentrations- und Vernichtungslager hervor. Aber eine substanzielle historische Beschreibung und Analyse der konkreten NS-Massenverbrechen und der »Endlösung der Judenfrage« erfolgte insgesamt betrachtet nicht.

Die erste Veröffentlichung zum Nationalsozialismus im Monat, also die Besprechung der Tagebücher des Propagandaministers des >Dritten Reiches $<$ vom britischen Labourpolitiker Crossmann aus dem Oktoberheft 1948 (vgl. IV.1.3) war überaus interessant. Und zwar vor allem durch die Tatsache, dass sie sozusagen zahlreiche weitere Beiträge vorwegnahm, die fortan zu diesem Thema im Monat erschienen und die für das in diesem Medium gezeichnete Bild von der NS-Herrschaft charakteristisch waren: Einerseits stellten die Ausführungen des Rezensenten insbesondere eine Personalisierung des >Dritten Reiches` dar, andererseits enthielt der Beitrag wiederum bemerkenswerte Reflexionen zum »ideologischen Täter« Goebbels sowie wichtige historische Fakten und Einschätzungen zum Nationalsozialismus. Beispielsweise wurde in der Veröffentlichung angesichts der Tagebucheintragungen des Propagandaminis- 
ters auf den Zusammenhang zwischen dem Verlauf des Zweiten Weltkriegs und der Judenvernichtung verwiesen sowie darauf, dass erst der Krieg dem NS-Regime die Möglichkeit verschaffte, die »Judenfrage« in seinem Sinne »in Angriff« zu nehmen. Zudem hob Crossmann Goebbels' entscheidendes persönliches Ziel hervor, nämlich, die europäischen Juden $\mathrm{zu}$ »liquidieren«. Denn obwohl sein Antisemitismus in der sogenannten Friedenszeit von der Taktik bestimmt gewesen war und er mithin eine »elastische Haltung« einnahm, war Goebbels für ihn letzten Endes ein ideologischer Überzeugungstäter. In den Augen von Crossmann war der NS-Propagandaminister »in seinem Antisemitismus ein ganz und gar linientreuer Prinzipienreiter«, da für ihn die Massenmorde vor dem Hintergrund des militärischen Verlaufes des Zweiten Weltkrieges in jedem Fall »zu Ende gebracht werden« mussten - und das »ohne Rücksicht auf den Widerstand der Kirchen und der Oberschicht«. In diesem Zusammenhang beleuchtete Crossmann die Frage, welche Personengruppe nach der »Ausschaltung der Juden« sozusagen als Nächstes an der Reihe gewesen wäre. Für ihn stand fest, dass Joseph Goebbels ein antibürgerlicher bzw. »echter Revolutionär« war und dass demzufolge für den NS-Ideologen die Aufgabe darin bestand, mit Entschlossenheit die »bürgerliche Klasse« endgültig zu vernichten, also die Industriellen, die Richter, die Aristokratie sowie das Offizierskorps, so Crossmann, »auszumerzen«.

Einen breiten Raum seiner Rezension nahm der Vergleich mit dem weltanschaulichen Gegner des Nationalsozialismus, dem sowjetischen Kommunismus unter Stalin, ein. Vor dem Hintergrund von Hitlers Ausführungen strich Crossmann besonders die »Stärke des Bolschewismus« sowie den »offenen Neid« heraus, die der deutsche »Führer« angesichts der sogenannten Vorteile auf der Seite Stalins sah. Dass dieser nämlich auf der Grundlage des felsenfesten Glaubens an die »bolschewistische Ideologie« bei den »bolschewistischen« Gewaltmaßnahmen nicht durch den Widerstand »einer Oberschicht« behindert würde, weil ebendiese »Opposition«, so Crossmann, bereits zu einem frühen Zeitpunkt getötet wurde. In seinen totalitarismustheoretisch inspirierten Ausführungen sprach er bemerkenswerterweise davon, dass Goebbels ein »Nationalbolschewist« war, der für seine Welteroberungspläne eine »marxistische Philosophie von der Macht« benutzte.

Genuine Einschätzungen und Reflexionen zum Aufbau des NS-Systems von Crossmann setzten besonders an der Stelle an, als in den Tagebucheintragungen die Sprache auf den für Deutschland negativen Kriegsverlauf kam. Auf dem Hintergrund des Hinweises auf die treue Gefolgschaft Goebbels' gegenüber Hitler enthielten die Ausführungen von Crossmann auch den Hinweis, dass im Verlauf des Jahres 1943 die SS der Wehrmacht sowie die Partei der Bürokratie überlegen war. Diese Feststellung kann implizit als eine gewisse Konkurrenzsituation dieser genuin nationalsozialistischen Herrschaftsinstitutionen interpretiert werden. Zudem konstatierte er - in Analogie zur Französischen und Russischen Revolution - beim »Robespierre der nationalsozialistischen Revolution«, also Goebbels, eine »Verachtung « für alle »Kollegen« aus dem NS-System, die »seinen Fanatismus« nicht teilten; ausgenommen wurden davon nur Hitler (für Goebbels die Verkörperung der >Mission der Deutschen`) und Himmler. In diesem Zusammenhang sprach Crossmann davon, dass es aufseiten von Goebbels (sowie Hitlers) einen Hass auf die Generäle der Wehrmacht gab. Vor diesem Hintergrund hatte Goebbels letzten Endes zu niemandem mehr Vertrauen, mit Ausnahme der SS, der Partei und dem »einfachen Volk«. Auch der Hinweis von Crossmann, dass ein Unterschied zwischen Goebbels und anderen sogenannten NS-Prominenten 
darin bestand, dass diese in den Augen von Goebbels in erster Linie an persönliche Interessen dachten, können so interpretiert werden, dass zwischen dem Propagandaminister und einzelnen führenden NS-Funktionären eine Konkurrenz herrschte.

Nach der Auswertung der Beiträge, in denen die Vorgeschichte der NS-Herrschaft thematisiert wurde (vgl. IV.1.4) kann festgestellt werden, dass in der Regel ein - mehr oder weniger - direkter Zusammenhang zwischen dem Versailler Vertrag und dem "Zusammenbruch« der Weimarer Republik einerseits sowie Hitlers Machtübernahme andererseits behauptet bzw. insinuiert wurde. So zum Beispiel im Kontext der Themenreihe "Versailles nach 35 Jahren« sowie dem 1959 erschienenen Beitrag Der letzte Friedensvertrag? Versailles nach vierzig Jahren von Sebastian Haffner. Auch wenn konzediert werden muss, dass in diesen Veröffentlichungen der inhaltliche Scheinwerfer nicht ausdrücklich auf das Ende der ersten deutschen Republik gerichtet wurde, können die Ausführungen der Autoren so interpretiert werden.

Zudem ergab die Auswertung, dass im Monat die tatsächlichen politischen, gesellschaftlichen und - wenn man so will - sozial- bzw. kollektivpsychologischen Folgen des Ersten Weltkrieges, die eine Voraussetzung für den Aufstieg des Nationalsozialismus zu einer Massenbewegung in den späten 1920er-Jahren darstellten, nicht ausdrücklich beschrieben und analysiert wurden. Nur Lüthy thematisierte in seinem Rezensionsaufsatz Der Führer persönlich. Gedanken beim Lesen zweier Biographien, d.h. der Besprechung der beiden Studien über Hitler, »nebenbei« den Aufstieg des »Trommlers« im Kontext der deutschen Verhältnisse nach dem Ende des Ersten Weltkrieges und dem als »Schmach« empfundenen Versailler Vertrag. Hier verwies er darauf, wie Hitler eine Versammlung der neugegründeten "Deutschen Arbeiterpartei« besuchte, die, so Lüthy, in München nur eine von zahlreichen radikal-nationalistischen Vereinigungen war und aus der später die NSDAP hervorging. In diesem Zusammenhang brachte Lüthy neben dem herrschenden völkischen Nationalismus in Teilen der deutschen Gesellschaft und der Rolle der Freikorps auch das facettenreiche politische und ideologische, explizit antisemitische und republikfeindliche Ressentiment zur Sprache, das Hitler als »Agitator" sozusagen ausbeutete. Zudem verwies er in seiner Rezension darauf, dass Hitler aus führenden Kreisen der Politik und Wirtschaft unterstützt wurde. Hitlers Aufstieg und spätere Machteroberung war nur möglich - so lassen sich seine Ausführungen lesen -, weil die erste deutsche Republik auf »dünnem Eis« errichtet wurde und nicht zuletzt Teile der deutschen Bevölkerung sowie führende Repräsentanten der politischen und ökonomischen Elite nicht bereit waren, die Weimarer Republik zu verteidigen, und sogar die Vorstellung hatten, Hitler als »bloßes Instrument« zu gebrauchen.

Indes: Diese und andere historische Details über die Vorgeschichte des Nationalsozialismus und den »Zusammenbruch « der Weimarer Republik waren in einer Veröffentlichung enthalten, deren eigentlicher Gegenstand die beiden Hitlerbiografien von Alan Bullock sowie von Walter Görlitz und Herbert A. Quint waren. Auf dem Hintergrund der Kategorisierung der Beiträge (IV.1.2) kann festgestellt werden, dass wichtige historische Details und genuin analytische Erkenntnisse zu dem Thema »Die Genesis der nationalsozialistischen `Machtergreifung〈. Oder: Das `Scheitern〈 der Weimarer Republik«im Monat insbesondere durch die Veröffentlichung von Lüthy erfolgten und folglich »indirekt bzw. nebenbei« gewonnen wurden. Darüber hinaus enthielten die weiteren aufgeführten Beiträge nur einzelne Informationen zur Vorgeschichte des Nationalsozialismus und konnten ebenfalls nur auf dem »indirekten Weg« gewonnen 
werden - wie zum Beispiel in dem Beitrag Lenin oder die Rolle des Einzelnen von Sidney Hook; in dem auf den sowjetischen Kommunismus bezogenen Beitrag konstatierte Hook qua totalitarismustheoretischer Perspektive, dass angesichts der Oktoberrevolution von 1917 führende Persönlichkeiten in Deutschland aus "Furcht vor dem Bolschewismus und vor einer Einführung des bolschewistischen Systems im Westen« Hitler unterstützten und zur Macht verhalfen.

Letztens Ende untersuchte die Zeitschrift Der Monat in einem einzigen Beitrag ausdrücklich die Vorgeschichte der nationalsozialistischen Machteroberung und mithin das »Scheitern« der Weimarer Republik. Nur die Veröffentlichung des Vorwortes des 1956 neuaufgelegten, zuerst 1946 erschienenen Buches Woran scheiterte die Republik von Weimar von dem Berliner CDU-Politiker Ferdinand Friedensburg versuchte ausdrücklich die konkreten Ursachen für den - wie der Autor schrieb - »unglückliche[n] Ausgang der ersten deutschen Demokratie« zu klären. Die Veröffentlichung von Friedensburg stellt durchaus eine differenzierte Auseinandersetzung dar. Gleichwohl drängte sich nach der Lektüre der Eindruck auf, dass seine Analyse des »Zusammenbruches« der Weimarer Republik aus einer rechtskonservativen Perspektive erfolgte, die zweifelsohne in Ansätzen ein gewisse Kontinuität von Vorurteilen anzeigte, die aus dem Arsenal der sogenannten konservativen Revolution stammten.

So stellte Friedensburg vor allem den alliierten Friedensvertrag von Versailles sehr stark in den Mittelpunkt seiner Beschreibung und Analyse der Ursachen für den Untergang der Weimarer Republik. Seiner Auffassung nach hatten der Versailler Vertrag und mithin die alliierten Staaten eine Mitverantwortung für die Etablierung der NS-Diktatur, insoweit er suggerierte, dass ein »vernünftiger und gerechter Friede« das erste demokratische Regime in Deutschland stabilisiert, gefestigt hätte. Ebendieses Vorurteil stammte aus der Weimarer Republik. Seinerzeit bekämpften die rechtsbürgerlichen Parteien mit dem Topos vom sogenannten Versailler Diktat die Verständigungspolitik der demokratischen Regierung und schufen damit explizit und implizit die Voraussetzungen für das »Bündnis« mit dem Führer der NS-Bewegung, das mit der Berufung Hitlers zum Reichskanzler seinen »krönenden« Abschluss fand. Das tatsächliche politische Ziel der rechtsbürgerlichen Parteien bestand seinerzeit primär in der Beseitigung der demokratischen Republik und in der Transformation in ein autoritäres System, das die Ausschaltung der demokratischen Linken möglich machen sollte. Hierbei war die radikale Kritik an den Vertragsverpflichtungen, d.h. der Kampf gegen »Versailles«, in den 1920er-Jahren nur ein Vorwand.

Darüber hinaus sah Friedensburg einen unmittelbaren Zusammenhang zwischen dem Versailler Vertrag und der deutschen Revolution von 1918, die er als ein Umsturzversuch der »kommunistischen Bewegung « apostrophierte und als »bedeutungsvolle Belastung« für das neue Staatswesen betrachtete; überhaupt war die Weimarer Republik ständig von der »radikalen Linken« bedroht. Andererseits war Friedensburg der Ansicht, dass das demokratische System in Bezug auf den innenpolitischen Gegner der Rechten von der - wie er schrieb - "gleichen Schwächlichkeit und Unentschlossenheit« gekennzeichnet war wie gegenüber der Linken. Gleichwohl konzedierte er, dass in den Institutionen der Verwaltung, der Polizei und der Justiz eine gewisse Nachsicht beispielsweise gegenüber der NSDAP konstatiert werden musste, die zum Teil eine »sträfliche geheime Sympathie« (unter anderem beim »Kapp-Putsch« 1920 und beim »Hitler-Putsch« 1923) bewiesen. 
Letztlich drängte sich nach dem Studium des Beitrages von Friedensburg der Eindruck auf, dass er von der Prämisse ausging, dass im Prinzip die Konstitution des demokratischen Systems sowie der dieses System repräsentierenden Parteien der politischen Klasse stabil war und stabil geblieben wäre, wenn man - abgesehen von den bereits genannten Gründen - nur frühzeitig vor allem die noch nicht allzu starke NSDAP in den 1920er-Jahren in die Regierungsverantwortung genommen hätte. Zudem war bemerkenswert, wie stark er besonders die persönliche Verantwortung von Reichspräsident Paul von Hindenburg für das Scheitern der Weimarer Republik respektive für den sogenannten Sturz der Republik hervorhob und dass erst vor diesem Hintergrund die »nationalsozialistische Revolution « gelingen konnte.

Friedensburg sprach in diesem Zusammenhang von einem »Sturz der Republik« bzw. von einer »nationalsozialistischen Revolution «. Tatsächlich avancierte Hitler am 30. Januar 1933 nicht aus eigener Kraft zum Reichskanzler. Hitler wurde aufgrund spezieller politischer und sozialer Strukturbedingungen der Weimarer Republik, deren Auflösung spätestens seit Beginn der 1930er-Jahre im Gang gekommen war, von der national-konservativen Machtgruppe um Reichskanzler Franz von Papen und Kurt von Schleicher - die als Gegner des parlamentarisch-demokratischen Systems einen autoritären Staat favorisierten - im Rahmen des »Zähmungskonzeptes« an die Macht gebracht, indem sie Hindenburg davon überzeugen konnten, Hitler zum Reichskanzler zu ernennen. Von den näheren historischen Umständen, die Hitlers Erfolg möglich machten, enthielt der Beitrag von Friedensburg keine konkreten Angaben. Beispielsweise wurden von ihm die ganz spezifischen atmosphärischen Bedingungen im Nachkriegsdeutschland nicht angesprochen, ohne die der Aufstieg des Nationalsozialismus in den 1920er-Jahren nicht möglich gewesen wäre - unter anderem ein militanter Nationalismus in breiten Schichten der deutschen Bevölkerung sowie ein grassierender Antisemitismus, die auch und vor allem die Grundlage bildeten für politisch-ideologische Entwürfe, die die Rettung Deutschlands und die nationale Wiedergeburt zum existenziellen Inhalt hatten. Zudem ging Friedensburg mit keinem Wort auf die tatsächlichen Wahlen in den 1920er- und zu Beginn der 1930er-Jahre ein. Insgesamt lag über Friedensburgs Ansatz, der das »Scheitern« der Weimarer Republik erklären sollte, ein »Schleier des Nebels«, der die komplexen Ursachen für den Aufstieg der nationalsozialistischen Massenbewegung, Hitlers Machteroberung sowie die »Auflösung« der ersten demokratischen Republik eher verdunkelte. Nicht zuletzt beim Aufzählen der »weiteren Gründe«, die zum sogenannten Sturz führten, zeigte sich in diesem Zusammenhang beim Autor eine skeptische Grundhaltung gegenüber dem demokratisch-parlamentarischen (Parteien-)System, aus der letzten Endes ein explizit elitentheoretischer Ansatz zum Ausdruck kam.

Auf der Grundlage der Auswertung der Beiträge zur innen- und außenpolitischen Entwicklung des Nationalsozialismus von Januar 1933 bis 1938 (vgl. IV.1.5) kann konstatiert werden, dass der Monat - abgesehen von der Veröffentlichung Der »völkische Rechsstaat «. Zur nationalsozialistischen Rechtspolitik von Hofer sowie einzelnen Bemerkungen und Einschätzungen Trevor-Ropers - nur in zwei Beiträgen auf diese zentrale Phase der NS-Herrschaftskonstituierung ausdrücklich einging: in Winfried Martinis Artikel zur »Röhm-Affäre« sowie in der Veröffentlichung von Rohan Butler zum »Fall Fritzsch«. Darüber hinaus thematisierte der Monat die innen- und außenpolitische Entwicklung Deutschlands nach der NS-Machteroberung und -konsoldierung bis zum Anschluss Österreichs 1938 nur noch »indirekt bzw. nebenbei«. Das heißt, auf 
die innen- und außenpolitische Entwicklung Deutschlands in diesen Jahren ging die Zeitschrift Der Monat nicht grundsätzlich ein. Insofern man sich mit der NS-Herrschaftspolitik in den Jahren 1933 bis 1938 auseinandersetzte, erfolgte dies - abgesehen von einer totalitarismustheoretischen Perspektive - entweder vor dem Hintergrund des »Hitler-Phänomens« (wie Lüthys Beitrag Der Führer persönlich) oder indem dies in erster Linie »indirekt bzw. nebenbei« erfolgte.

Was man nämlich im Monat im Hinblick auf die politischen Folgen in der Regel »indirekt bzw. nebenbei« erfuhr, die mit dem historischen Datum des 30. Januar 1933, d. h. der »Machtergreifung (Hofer), verbunden war, war die Tatsache, dass nicht wenige tatsächliche Gegner (Sozialdemokraten und Kommunisten) des Nationalsozialismus sowie (Links-)Intellektuelle, Schriftsteller, Künstler und deutsche Juden, die sich der drohenden persönlichen Gefahr gewahr wurden, Deutschland verließen und in die Emigration gingen. Mit dem konkreten NS-Terror, der nach der Machteroberung einsetzte, setzte man sich indes nicht ausdrücklich auseinander. In diesem Zusammenhang ging die Zeitschrift auch nicht auf den Aufbau des Konzentrationslagersystems in den ersten Jahren der NS-Herrschaft - abgesehen von einzelnen Hinweisen wie zum Beispiel von Hermann Kesten oder auch Hans Sahl - ausdrücklich ein, sodass letztlich ausschließlich der NS-Experte Hofer dieses Thema im Monat in einem einzigen Beitrag näher beschrieb und analysierte.

In dem Beitrag zur »Röhm-Affäre« von Martini aus dem Jahre 1957 war auffallend, dass der Autor im Vergleich zu dem Aufsatz Die »Zweite Revolution« - Der 30. Juni 1934 von Hermann Mau, der in den Vierteljahresheften für Zeitgeschichte des Münchener Institutes für Zeitgeschichte im Jahre 1953 veröffentlicht wurde und der - wie erwähnt die eigentliche Grundlage für seinen Aufsatz bildete, im Hinblick auf die Analyse der Mordaktion eine andere historische Interpretation vornahm; obwohl Martini sich hierbei auf die von Mau präsentierten historischen Fakten und sich zudem durchaus in Ansätzen dessen Deutungsangebot zu eigen machte. Abgesehen von dem verharmlosenden Bild, das Martini von den beiden beteiligten SS-Tätern der Mord- und Verhaftungsaktion am 30. Juni 1934, also Michael Lippert und Sepp Dietrich, zeichnete, ließen sich einige Auffälligkeiten feststellen:

1. So nahm der Autor primär einen genuin personenzentrierten Blick auf die »Röhm-Affäre« ein, obwohl er zweifellos eine Konkurrenz zwischen SA und Reichswehr konzedierte. In seinem Artikel war im Gegensatz zu der Veröffentlichung von Mau von der existierenden institutionellen Rivalität zwischen der NSDAP und der SA nicht die Rede, obwohl sie von Anbeginn der NS-Bewegung charakteristisch war. Zudem thematisierte Martini nicht den fundamentalen Konflikt zwischen der SA und der SS. Zweifelsohne war die SS im Jahre 1934 noch in der SA unter der Führung von Röhm eingegliedert. Jedoch nutzte insbesondere Himmler die Ausschaltung Röhms und der SA dafür, um die SS zu einer entscheidenden Säule im NS-Machtsystem werden zu lassen. Der Aufstieg Himmlers und der SS nach dem »Röhm-Mord«, die kurz darauf, am 20. Juli 1934, von Hitler zu einer selbstständigen, de facto nur dem »Führer« unterstellten Organisation ernannt wurde, hatte für die weitere Entwicklung des Nationalsozialismus eine fundamentale Bedeutung, ohne dass Martini darauf einging.

2. So beleuchtete der Verfasser in diesem Zusammenhang die Rolle Hitlers in der »Röhm-Affäre« im Grunde genommen nicht, und insofern wurden die zentralen Hintergründe für die Ausschaltung des potenziellen Rivalen Röhm und der SA für Hitlers politische Ziele nicht angesprochen. Hitler konnte durch die Mordaktion seine Stel- 
lung im NS-Staat zementieren und zudem einen grundsätzlichen Machtgewinn verzeichnen.

3. So schrieb Martini seine historische Interpretation der Nacht der langen Messer auch und vor allem aus einer quasi totalitarismustheoretischen Perspektive heraus, weil er hinsichtlich seiner Beurteilung der SA und ihrer politischen Ziele und Motivation explizit eine Analogie zur Oktoberrevolution von 1917 herstellte, als er unter anderem Röhm als den »Träger des NS->Trotzkismus« «klassifizierte.

4. Auffallend war auch, dass Martini am Ende seines Artikels insinuierte, dass Rudolf Diels gewissermaßen für die "gute Seite« des Nationalsozialismus stand, der mit dem »30. Juni 1934« absolut nicht einverstanden war und sich post festum radikal von der »Einführung des Mordes als staatliches Prinzip« distanzierte. In diesem Zusammenhang wäre es bei einem um tatsächliche Aufklärung bemühten Autor - wie zum Beispiel Hermann Mau - wichtig gewesen, die Rolle von Diels beim In-Gang-Kommen der NS-Terrorpolitik (unter anderem beim Aufbau der Konzentrationslager) zu thematisieren, was Martini unterließ.

5. Zudem ging Martini nicht darauf ein, dass neben führenden Vertretern der SA auch einige Gegner des Nationalsozialismus bzw. "Erzfeinde« von Hitler ermordet wurden: zum Beispiel Gregor Strasser, General Kurt von Schleicher, der ehemalige bayerische Generalstaatskommissar von Kahr oder auch der konservative Publizist Edgar Jung.

Wenn oben konstatiert wurde, dass der Monat auf die innenpolitische Entwicklung in Deutschland nach der Ernennung Hitlers zum Reichskanzler Anfang 1933 bis 1938 nicht grundsätzlich einging (mit Ausnahme der Beiträge von Hofer und Martin sowie von Butler zum »Fall Fritzsch«), so galt dies infolgedessen auch für die »Judenpolitik«. Bis auf vereinzelte Hinweise, die von Autoren »indirekt bzw. nebenbei« gemacht wurden, weil sie in ihren Veröffentlichungen beispielsweise die Flucht von jüdischen Mitbürgern ansprachen, ging die Zeitschrift letzten Endes auf die antijüdische Politik des NS-Herrschaftssystem ausschließlich im Kontext des Pogroms vom 8./9. November des Jahres 1938 ein. Hierbei kann indes von einer genauen Beschreibung und Analyse der antisemitischen Ausschreitungen nicht gesprochen werden, weil es sich auch in diesen Fällen um Hinweise handelte, die »indirekt bzw. nebenbei« erfolgten. Das heißt, sowohl eine substanzielle Auseinandersetzung als auch eine grundsätzliche historisch-politische Bewertung der »Judenpolitik« des NS-Systems in den Jahren 1933 bis 1938 erfolgte nicht.

Aufgrund der empirischen Auswertung des Monat im angegebenen Zeitraum kann festgestellt werden, dass sich die allermeisten Veröffentlichungen zur Geschichte des >Dritten Reiches` mit dem Themenkomplex Zweiter Weltkrieg, militärisch-konservativer Widerstand gegen die "Hitler-Diktatur« sowie der »Memoirenliteratur schäftigten (vgl. IV.1.6 sowie IV.1.7). Denn mit knapp 30 Beiträgen bildeten der Zweite Weltkrieg, der deutsche Widerstand sowie die »Memoirenliteratur « den eigentlichen Schwerpunkt bei der ausdrücklichen Auseinandersetzung mit der Geschichte des >Dritten Reiches<. Hierbei wurde auf den individuellen und organisierten sozialdemokratischen, kommunistischen oder jüdischen Widerstand gegen die NS-Herrschaft nicht explizit eingegangen. Die Veröffentlichung Die verhinderten Hochverräter. Wege und Irrwege neuerer deutscher Memoirenliteratur von de Mendelssohn wurde hierbei insbesondere aus zwei Gründen ausführlich referiert: Einerseits stellte die Veröffentlichung des Emigranten de Mendelssohn in Bezug auf die Analyse der moralischen und 
politischen Motive des deutschen Widerstandes des »20. Juli« die »schärfste« Auseinandersetzung dar, die sich auch in zahlreichen, allerdings größtenteils von de Mendelssohns Beitrag ausdrücklich distanzierenden Leserbriefreaktionen niederschlug; andererseits nahm der Autor seinen auf hohem Niveau geschriebenen Rezensionsaufsatz zum Anlass, in allererster Linie auf das »Erinnerungsbuch« von Ernst von Weizsäcker einzugehen.

Im Zusammenhang mit dem Zweiten Weltkrieg wiederum kann festgestellt werden, dass die Zeitschrift insbesondere die diplomatische Vorgeschichte des Zweiten Weltkrieges ausdrücklich in das Zentrum des (Erkenntnis-)Interesses rückte, und zwar durch die (Vorab-)Veröffentlichung der vierteiligen Reihe Diplomatie auf schiefer Bahn von Joachim G. Leithäuser im Jahre 1952. An der im darauffolgenden Jahr in Buchform erschienenen wissenschaftlichen Studie war bemerkenswert, dass der Autor die nationalsozialistische bzw. Hitlers Außenpolitik der Jahre 1938 bis 1939, d.h. vom »Anschluss« Österreichs bis zum Überfall der Wehrmacht auf Polen, zum einen substanziell untersuchte und zum anderen diese Untersuchung auf der Grundlage einer umfassenden zeitgenössischen Forschungsliteratur sowie breiten Dokumentenbasis durchführte, die zudem im Monat insgesamt mitabgedruckt wurde. Mithin ging das Zeitschriftenorgan in extenso unter anderem auf die neuartigen diplomatischen respektive außenpolitischen Methoden Hitlers ein, die vor allem aus einem Ineinandergreifen von Erpressung, Lügen, Propaganda, Gewalt und Einschüchterung bestand. Die wissenschaftliche Studie von Leithäuser untersuchte die totalitäre Außenpolitik Deutschlands indes nur für den Zeitraum der »unblutigen Eroberungen« des NS-Herrschaftsregimes. Infolgedessen wurden in der Studie die insbesondere nach dem Überfall der Wehrmacht auf die Sowjetunion in Gang gekommenen Massenverbrechen des NS-Systems nicht in den Blick genommen und auch in diesem Zusammenhang die weltanschaulichen Aspekte der außenpolitischen Ziele des »Hitlersystems« nicht beleuchtet.

Dass der Zweite Weltkrieg auch durch »die« nationalsozialistische Weltanschauung motiviert war und einen im Vergleich zum Ersten Weltkrieg qualitativen Unterschied aufwies, stellte der englische Historiker Rohan Butler fest. Im Rahmen der Rezension des ersten Bandes der Studie Der Zweite Weltkrieg 1939-1945 vom deutschen Historiker Walter Görlitz insistierte Butler zudem auch und vor allem auf die wirtschaftliche Seite des Krieges und hob bei seinen analytischen Reflexionen erstens die für ihn offensichtlich fehlende "weitblickende Planung und Koordination« des angeblichen »Führerstaates« hervor, zweitens die Herrschaftsmethode Hitlers, nämlich mit dem Ernennen von sogenannten Sonderbevollmächtigten zu agieren, sowie drittens, dass eben diese Herrschaftsmethode einen "schier unentwirrbaren Kompetenzkampf«im Nationalsozialismus zur Folge hatte. Seine Rezension nahm Butler zudem ausdrücklich zum Anlass für eine Kritik an Görlitz, weil dieser in seiner Untersuchung die seiner Meinung nach aus wirtschaftlichen Gründen erfolgte Zwangsarbeit von »Fremdarbeitern« nicht zur Sprache brachte und auch die deutschen Konzentrationslager sowie die NS-Verbrechen nicht thematisierte.

Nähere Einzelheiten über den »Vernichtungskrieg« (Butler) sowie über die ideologischen Grundlagen der nationalsozialistischen Lebensraumpolitik im Hinblick auf das »Unternehmen Barbarossa« enthielt - neben den Veröffentlichungen aus Arendts Totalitarismusstudie sowie ihrem Rezensionsaufsatz Bei Hitler zu Tisch - der dreiteilige Beitrag Der Fall Wlassow von George Fischer, der allerdings im Monat zur 
(wissenschaftlichen) Untersuchung des sowjetischen Kommunismus erschien. Der amerikanische Autor warf beispielsweise in seiner Studie zum »Fall Wlassow« im Zusammenhang seiner Ausführungen über die ideologischen Hintergründe der »Ostpolitik des Dritten Reiches« den Scheinwerfer auf den wirtschaftlichen Ausbeutungscharakter des Krieges (u.a. Rohstoff- und Lebensmittelgewinnung in der Ukraine sowie Zwangsarbeit von sowjetischen Gefangenen) sowie auf die rassistische Vorstellung vom "slawischen Untermenschen«, die Fischer zufolge auch und vor allem mit den jüdischen Bewohnern der Sowjetunion assoziiert wurde. In den Augen von Fischer wurden die Massenverbrechen nicht zuletzt von den "SS-Einsatzkommandos" begangen, die im Verlauf der Besatzungspolitik »immer brutalere Formen annahmen«, die indessen hinsichtlich der Grausamkeit, so der Autor, »vielleicht nur von den Grausamkeiten des sowjetischen NKWD übertroffen wurden«.

Zudem enthielt der Beitrag auch insofern einen analytischen Blick auf das NS-System, als Fischer nämlich in Ansätzen konkret auf die verschiedenen Interessen und politischen Vorstellungen von führenden Funktionären und auf genuine Institutionen des NS-Systems im Rahmen der "Ostpolitik« einging. Mithin stellte er implizit die Vorstellung infrage, dass es sich beim Nationalsozialismus um ein Herrschaftssystem handelte, das ausschließlich vom Primat der »Führerentscheidungen« abhing und als ein »Hitlerismus« zu erklären sei. Genauer gesagt: Fischer strich im Kontext seiner Ausführungen über den NS-Vernichtungskrieg gegen die Sowjetunion einerseits die hohe Bedeutung von einzelnen NS-Funktionären heraus - wie dem Reichskommissar für die Ukraine Erich Koch oder dem Reichsminister für die besetzten Ostgebiete Alfred Rosenberg - und andererseits, dass führende NS-Funktionäre ganz verschiedene Vorstellungen und Interessen im Kontext der deutschen Besatzungs- und Gewaltpolitik in Südosteuropa und speziell beim »Fall Wlassow« hatten. Infolgedessen konnte er auch feststellen, dass beim »Unternehmen Barbarossa« in der NSDAP sehr wohl »extreme Elemente existierten, die die deutsche Politik mitbestimmten bzw. mitbeeinflussten. Kurzum: Die Veröffentlichung Der Fall Wlassow lieferte nähere Einzelheiten zum NS-Herrschaftssystem, nämlich zu verschiedenen genuinen NS-Institutionen sowie führenden NS-Funktionären, sodass in diesem Zusammenhang nicht nur die bekannten »Größen« des Nationalsozialismus um Hitler (Göring, Himmler oder Goebbels) in den Fokus des (Erkenntnis-)Interesses gerieten. Insofern stellte dieser Beitrag für die »Analyse des Nationalsozialismus« im Monat eine Ausnahme dar. Indes: Der Beitrag von George Fischer erschien in der Zeitschrift als eine wissenschaftliche Untersuchung über den sowjetischen Kommunismus und nicht zur Geschichte des >Dritten Reiches`.

Auf die Wehrmacht und die Einsatzgruppen beim »Unternehmen Barbarossa«kam Görlitz in seiner Besprechung des Buches von Hans Laternser, dem Verteidiger von führenden Wehrmachtsgenerälen bei den Nürnberger Prozessen, zu sprechen, ohne allerdings hierbei die spezielle Rolle der Einsatzgruppen im Zweiten Weltkrieg näher zu klären. Insgesamt blieben seine Ausführungen und Reflexionen hinsichtlich der historischen Bewertung des NS-Herrschaftssystems sehr marginal. Dem Autor war es in erster Linie um eine radikale Kritik an den Nürnberger Prozessen und seinen zugrundeliegenden Prinzipien zu tun. Gleichwohl enthielt der Beitrag bemerkenswerte Feststellungen. So vertrat Görlitz die Ansicht, dass die deutsche Geschichtsschreibung, die sich mit dem Zweiten Weltkrieg beschäftigte, unter anderem unter dem »Fehlen der Akten« litt. Zweitens konstatierte er ausdrücklich, dass die Vorstellung einer 
»straffen Gliederung« des hitlerschen »Führerstaates« ein zu eindimensionales Bild darstelle. Denn der »Führerstaat« bestand seiner Analyse zufolge aus einem »Gewirr von Stäben, sich überschneidenden Führungsbereichen und einander auf das heftigste befehdenden Machthabern (und) glich im Grunde einer verhüllten diktatorischen Anarchie«.

Die insbesondere nach dem deutschen Angriff am 22. Juni 1941 auf die Sowjetunion in Gang gekommenen nationalsozialistischen Massenverbrechen (vgl. IV.1.8) wurden im Monat zweifelsohne nicht nur als historisches Faktum zur Sprache gebracht, sondern auch mit Blick auf die Vernichtung der europäischen Juden von mehreren Autoren in der qualitativen und quantitativen Bedeutung ausdrücklich hervorgehoben. Allerdings erfolgte keine substanzielle Auseinandersetzung mit dem Komplex der deutschen Gewaltverbrechen und der »Endlösung der Judenfrage«. Mit anderen Worten: Eine konkrete Untersuchung respektive Auseinandersetzung mit der Vernichtungsdimension des NS-Herrschaftssystems und der beteiligten Akteure, Gruppen und Institutionen wurde nicht vorgenommen. Letzten Endes fokussierte Der Monat ebendieses facettenreiche Thema im Kontext des hitlerzentrierten Bildes des Nationalsozialismus oder zumeist qua totalitarismustheoretischer Perspektive und Erkenntnisse auf die unmittelbare NS-Führungsgruppe um Hitler, Göring, Goebbels, Himmler sowie dem abstrakt bleibenden SS-Apparat. Zudem wurden in der Zeitschrift auch die weltanschaulichen Aspekte und Hintergründe der nationalsozialistischen Massenverbrechen, also der Zusammenhang von Ideologie und Terror, nicht ausdrücklich beleuchtet.

Die beiden Beiträge von Eugen Lerch (Der Rassenwahn. Von Gobineau zur UNESCOErklärung) und Melvin Richter (Der Begriff der Rasse. Aus dem Briefwechsel zwischen Tocqueville und Gobineau) stellten eine indirekte Thematisierung der deutschen Massenverbrechen und der NS-Ideologie dar. Obwohl insbesondere der Beitrag von Lerch ansatzweise auch auf die tatsächliche nationalsozialistische »Judenpolitik" sowie den Konnex von "Theorie und Praxis« einging, enthielten insgesamt betrachtet beide Veröffentlichungen primär ideengeschichtliche Reflexionen zur antisemitischen Rassentheorie des Nationalsozialismus und setzten sich mit den konkreten deutschen Massenverbrechen und mit der »Endlösung der Judenvernichtung« nur »nebenbei« auseinander.

Nach der empirischen Auswertung der einzelnen Ausgaben des Monat von Oktober 1948 bis April 1960 kann festgestellt werden, dass letzten Endes ausschließlich in vier Veröffentlichungen eine ausdrückliche und empirisch ausgerichtete Thematisierung und Auseinandersetzung mit den NS-Massenverbrechen sowie der Vernichtung der europäischen Juden erfolgte. Um folgende Beiträge handelte es sich, die mit Blick auf ebendiesen Verbrechenskomplex ausführlich referiert wurden:

1. Friedrich Carl Westphal: Noch einmal »Die verhinderten Hochverräter«; hierbei handelte es sich um einen Leserbrief zu dem Beitrag Die verhinderten Hochverräter. Wege und Irrwege neuerer deutscher Memoirenliteratur von Peter de Mendelssohn (Mai 1951),

2. Hannah Arendt: Bei Hitler zu Tisch (Oktober 1951),

3. Herbert Lüthy: Der Führer persönlich. Gedanken beim Lesen zweier Biographien (November 1953),

4. Walter Laqueur: Die Affäre Kastner (September 1955). 
Wie in den Ausführungen in Kapitel IV.1.8 bereits konstatiert wurde, konzentrierten sich Lüthy und Arendt aufgrund des (Untersuchungs-)Gegenstandes ihrer Rezensionsaufsätze auf die Biografie Hitlers bzw. auf das »Hitler-Phänomen«. Beide Veröffentlichungen bestachen durch den Versuch, Hitler rational zu erklären und ihn nicht als »Dämon« zu beschreiben und zu interpretieren. Beide Beiträge enthielten auch substanzielle analytische Reflexionen und Deutungen über die genuin nationalsozialistische Weltanschaung Hitlers. Auf der Basis zeitgenössischer Forschungserkenntnisse und der zum damaligen Zeitpunkt vorhandenen Quellen war für Lüthy und Arendt Hitlers Weltanschauung eine entscheidende Voraussetzung für die ihrer Auffassung nach durch den »Führerbefehl« in Gang gekommenen Massenverbrechen insbesondere an den europäischen Juden. Beide Autoren warfen einen Blick auf den tatsächlichen Zusammenhang zwischen Ideologie und Terror. Zudem ging aus beiden Veröffentlichungen hervor, dass neben der millionenfachen Ermordung von europäischen Juden, der »Endlösung der Judenfrage« (Lüthy), ebenfalls durch einen »Führerbefehl« mit dem Überfall auf die Sowjetunion das »Euthanasieprogramm« zur Ausrottung der sogenannten Minderwertigen und Geisteskranken durchgeführt wurde. Dieser persönliche Befehl von Hitler wurde hierbei von Arendt bereits auf den 1. September 1939 datiert.

Die beiden Veröffentlichungen von Lüthy und Arendt zum >Dritten Reich zählten $\mathrm{zu}$ den wenigen Beiträgen, in denen ein genuin analytischer Blick auf das NS-Herrschaftssystem und seiner Gewaltverbrechen geworfen wurde. Insofern nämlich, als hier im Kontext der Ausführungen zum ideologisch motivierten rassistischen Lebensraum- und Eroberungskrieg in den südosteuropäischen Ländern beispielsweise das sogenannte Rasse- und Siedlungsamt des Reichsführers SS unter der Führung von Himmler ins Blickfeld geriet. Zudem war es Arendt, die in diesem Zusammenhang ausführlich auf die sogenannten Einsatzgruppen einging. Unter sämtlichen Autoren des Monat, die im Laufe der Jahre zum »Führerstaat« schrieben, war sie die Einzige, die in ihrem empirisch ausgerichteten Beitrag Bei Hitler zu Tisch auf die zentrale Rolle der Einsatzgruppen im Kontext der »Endlösung der Judenfrage« einging. Arendt wies unter anderem darauf hin, dass die vier berüchtigten Einsatzgruppen nur wenige Wochen vor dem Angriff auf die Sowjetunion gebildet wurden und ausdrücklich für die "Ausmerzungsarbeiten«, d. h. für die »Ausrottung des Judentums«, vorgesehen waren. Vor dem Hintergrund der »beeidigten« Berichte der »Führer der Einsatzgruppen«, so Arendt, konnte sie unter anderem die Feststellung treffen, dass bereits im Sommer 1941 über »dreihunderttausend Juden durch Massenerschießungen umgekommen waren«.

Neben Lüthy und Arendt ging der Leser des Monat, Friedrich Carl Westphal, auf den Hintergrund der Rolle des Auswärtigen Amtes und speziell des Staatssekretärs Ernst von Weizsäcker, ebenfalls grundsätzlich auf die Vernichtung der europäischen Juden ein. Insbesondere auf der Grundlage des Buches Das Urteil im Wilhelmstraßenprozeß vom stellvertretenden Hauptankläger in Nürnberg, Robert Kempner, konnte Westphal in seinem Leserbrief den Nachweis vom komplexen Vernichtungsprozess der europäischen Juden durch den NS-Staat erbringen. Denn durch den Leserbrief von Westphal wurde - unter anderem durch die von ihm herangezogenen, im Wilhemstraßenprozess auf Grundlage empirischer Quellen zutage geförderten gesicherten Erkenntnisse - explizit und implizit die Erkenntnis vermittelt, dass die "Endlösung der Judenfrage« nicht von einigen wenigen führenden NS-Funktionären (Hitler, Gö- 
ring, Himmler etc.) zu verantworten war und ausschließlich von der SS und den Einsatzgruppen begangen wurden (die ihre Taten im »Schatten« der südosteuropäischen Länder durchführten und »verheimlichten«). Westphal konnte in nuce zeigen, dass die deutschen Massenverbrechen letzten Endes vom NS-System begangen wurden, in das führende staatliche Institutionen in Deutschland und in den besetzten Ländern beteiligt waren. Westphal konstatierte in diesem Zusammenhang nämlich unter anderem.

1. Die zentrale Rolle des Auswärtigen Amtes und führender Mitarbeiter wie zum Beispiel des Staatssekretärs von Weizsäcker bei der »Endlösung der Judenfrage«.

2. Dass das Auswärtige Amt regelmäßig die sogenannten Einsatzgruppenberichte mit ausdrücklichen Informationen über die Massenmorde an der jüdischen Bevölkerung in den von der Wehrmacht besetzten südosteuropäischen Ländern, aus denen detailliert hervorging, wie ganze Gebiete »judenfrei« gemacht wurden, nicht nur zur Kenntnis nahm, sondern zudem viele dieser Berichte von Weizsäcker und Wörmann auch abgezeichnet wurden.

3. Dass das Auswärtige Amt mit seinen Fachbeamten bei der Deportation von Juden in zahlreichen vom Nationalsozialismus besetzten Ländern in Europa einen maßgeblichen Anteil hatte und mithin ein »Mitwirkungsorgan des Naziterrors« (Westphal) wurde.

4. Dass bei der »Endlösung der Judenfrage« nicht nur die SS und die Einsatzgruppen, sondern zudem Institutionen wie die Gestapo, die Polizei oder das Auswärtige Amt beteiligt waren.

Die Veröffentlichung Die »Affäre Kastner«. Wo liegt die Grenze zwischen Heldentum und Verrat? von Laqueur stellte im Kontext der Analyse des Nationalsozialismus im Monat insofern eine Ausnahme dar, weil hier bei der Thematisierung der Ermordung der europäischen Juden auf dem Hintergrund der Deportation der ungarischen Juden auch die nationalsozialistischen Konzentrations- und Vernichtungslager ausdrücklich in den Mittelpunkt gerückt wurden. Hierbei vermittelte Laqueur detaillierte Informationen über den Umfang der täglichen Transporte der ungarischen Juden nach Auschwitz. Sein Hinweis, dass in den letzten Kriegsmonaten täglich 12000 Juden aus Ungarn, »Männer und Frauen, Greise und Kinder«, dem Vernichtungsprozess zugeführt wurden und dass das \-Dritte Reich « das Vernichtungslager Auschwitz-Birkenau errichtete, um den »neuen Zustrom zu bewältigen«, kann ohne Zweifel auch als ein wesentlicher Beitrag zur konkreten Beschreibung und Analyse des Nationalsozialismus und seiner Gewaltverbrechen im Monat angesehen werden. Zudem enthielt der Beitrag von Laqueur einerseits ausdrückliche Hinweise auf verantwortliche SS-Funktionäre bei der Deportation und Vernichtung der ungarischen Juden, wie zum Beispiel Becher und Wisliczeni. Andererseits wurde die Schlüsselrolle von Eichmann beim Vernichtungsprozess der ungarischen Juden angesprochen, ohne allerdings hierbei seine zentrale Aufgabe bei der »Endlösung« insgesamt genauer zu beleuchten sowie die besondere Rolle von dem »Büro Eichmann« beim Ingangkommen des Vernichtungsprozesses der ungarischen Juden zu analysieren. Daraus folgte auch, dass letzten Endes Eichmann im Grunde genommen »nur« als »Judenspezialist der Nazis« charakterisiert wurde, ohne auf die besonderen Aufgaben des SS-Funktionärs als Leiter des »Judenkommandos« im RSHA näher einzugehen; ganz zu schweigen davon, dass in diesem Kontext die zentrale Bedeutung des RSHA im NS-Herrschaftssystem und speziell bei den NS-Massenverbrechen nicht näher thematisiert und mithin geklärt wurde. 
Gleichwohl vermittelte Laqueurs Artikel für die hier interessierende »Analyse des Nationalsozialismus« die wichtige historische Erkenntnis, dass Himmler und Eichmann als SS-Funktionäre aus unterschiedlichen Gründen in der Schlussphase des Zweiten Weltkrieges die Möglichkeit hatten, ungarische Juden zu retten, und ausdrücklich gegen Entscheidungen Hitlers agieren konnten. Insofern kann festgestellt werden, dass Laqueurs Beitrag das in der Regel im Monat fast ausschließlich auf Hitler fokussierte Bild des NS-Herrschaftssystems, das sich ausschließlich durch »Führerbefehle« konstituierte und auf dieser Grundlage funktionierte und was sich in den immer wieder verwendeten Kategorien wie »Führerstaat«, »Hitler-Staat«, »Hitlerismus« oder auch »Hitler-Diktatur« niederschlug, implizit infrage stellte.

$\mathrm{Zu}$ den Repräsentanten eines genuin personen- und hitlerzentrierten (Forschungs-)Ansatzes im Hinblick auf die Analyse des Nationalsozialismus im Monat gehörten auch Trevor-Roper und Hofer. Die angesichts der Auswertung der Hefte 1 bis 139 als NS-Experten des Monat kategorisierten prominenten internationalen Zeithistoriker vertraten in ihren Zeitschriftenbeiträgen (sowie in ihren in der deutschen Öffentlichkeit sehr populären Buchveröffentlichungen) den Standpunkt, dass Hitler der alles entscheidende Dreh- und Angelpunkt des Nationalsozialismus war. Letzten Endes vertraten Trevor-Roper und Hofer die Auffassung, dass Hitler im Prinzip alle für die innen- und außenpolitische Entwicklung des Nationalsozialismus wesentlichen Entscheidungen persönlich traf, was sich in den immer wieder kehrenden Begriffen »Führerentscheidungen« oder »Führerbefehle« bei der historischen Untersuchung der deutschen Geschichte von 1933 bis 1945 ausdrückte. In ihren Augen existierte der Nationalsozialismus im Grunde genommen fast ausschließlich aus der Person Hitler, sodass die beiden NS-Experten ständig die Kategorien »Hitlerismus«, »Hitlersystem«, »Führerstaat« oder »Hitler-Diktatur« verwendeten, um das nationalsozialistische Herrschaftssystem zu charakterisieren bzw. zu analysieren.

Die Auswertung des Monat ergab, dass sich die meisten Beiträge von Trevor-Roper und Hofer schwerpunktmäßig mit dem Zweiten Weltkrieg und dem deutschen Widerstand vom 20. Juli 1944 befassten, sodass infolgedessen Hitler a priori im Zentrum ihrer Auseinandersetzung mit dem Nationalsozialismus stand. Nur in drei Beträgen der beiden Mitarbeiter der Zeitschrift zum Thema Nationalsozialismus stand nicht ausschließlich Hitler im Zentrum des (Erkenntnis-)Interesses: nämlich in Himmlers Leibarzt und Martin Bormann von Trevor-Roper sowie in Der »völkische Rechtsstaat«. Zur nationalsozialistischen Rechtspolitik von Hofer. Allerdings dominierte in diesem Zusammenhang wiederum explizit und implizit eine totalitarismustheoretische Perspektive bei der Analyse des NS-Herrschaftssystems.

Insbesondere bezüglich Trevor-Roper kann festgestellt werden, dass der den inhaltlichen Scheinwerfer auf einzelne NS-Funktionäre richtete, die aufgrund ihrer institutionellen Funktion im NS-System auch im Kontext der zeitgenössischen totalitarismustheoretischen Diskussion eine zentrale analytische Bedeutung besaßen - nämlich Himmler und Bormann aus der unmittelbaren Führungsgruppe um Hitler. Überhaupt war auffallend, dass Trevor-Roper in seinen Veröffentlichungen zum »Führerstaat« immer wieder einen Vergleich zum sowjetischen Kommunismus unter Stalin zog und eine undifferenzierte Parallele zwischen dem Nationalsozialismus und dem Stalinismus suggerierte - zum Beispiel in seinem frühen Beitrag Die Generäle verteidigen sich mittels der Kategorien »jakobinische Diktatur« und »Diktatur 
des deutschen Proletariats« zur Beschreibung und Klassifizierung der sogenannten Herrschaft Hitlers in Deutschland.

Vor dem Hintergrund der zeitgenössischen Forschungserkenntnisse zur Geschichte des >Dritten Reiches kann mit Blick auf einzelne Beiträge der beiden NS-Experten im Monat konstatiert werden, dass mit Ausnahme der Veröffentlichungen über Martin Bormann von Trevor-Roper und Der »völkische Rechtsstaat« von Hofer letzten Endes keine substanziellen Erkenntnisse zu einer Analyse des NS-Herrschaftssystems enthalten waren. Die Genesis des NS-Herrschaftsregimes und die konkrete »Judenpolitik« in den Jahren 1933 bis 1945 und speziell die deutschen Massenverbrechen, mit dem Kernereignis der »Endlösung der Judenfrage«, hatten zudem keinen grundsätzlichen Stellenwert bei beiden.

In den Veröffentlichungen Trevor-Ropers spielte insbesondere der Aufstieg der nationalsozialistischen Massenbewegung in der Weimarer Republik, die Machteroberung und -konsolidierung sowie die Politik des Regimes im Verlauf der 1930er-Jahre bei der Thematisierung der Geschichte des >Dritten Reiches` keine Rolle. Seine Auseinandersetzung mit dem Nationalsozialismus fokussierte mutatis mutandis den Zeitraum des Zweiten Weltkrieges bis zur totalen politischen und militärischen Niederlage Deutschlands, d. h. die Jahre 1939 bis 1945. Insgesamt betrachtet, schrieb er über "populäre« Themen: zum Beispiel Hitlers letzte Tage, Lügen um Hitlers Leiche oder die Memoirenliteratur. In erster Linie stand Hitler im Zentrum. Mit Ausnahme von Martin Bormann kann von keiner anderen Veröffentlichung gesagt werden, dass sie genuin analytische Reflexionen und Einschätzungen zum NS-Herrschaftssystem enthielten. So kam der englische Historiker beispielsweise in Himmlers Leibarzt ausführlich auf Himmler zu sprechen, ohne indes speziell auf seine Rolle und genaue Funktion als Reichsführer SS und Chef der Gestapo im NS-Herrschaftssystem einzugehen. Dass Himmler qua Funktion über den gesamten Exekutivapparat herrschte und mithin zweifelsohne einer der mächtigsten Personen des NS-Herrschaftssystems war - zumal er auch noch 1943 zum Reichsinnenminister und 1944 zum Befehlshaber des Ersatzheeres aufstieg -, kam von Trevor-Roper nicht zur Sprache. Anders gesagt: In der Besprechung des Buches Totenkopf und Treue von Kersten war über Himmler in seiner Funktion als Reichsführer SS und Chef der gesamten deutschen Polizei, also einer spezifischen und mächtigen NS-Institution, nicht eigentlich die Rede. Trevor-Roper reduzierte letzten Endes Himmler zu einem reinen Befehlsempfänger von Hitler, ohne dass grundsätzliche analytische Aussagen über Himmler als den Herrscher eines genuinen Machtzentrums - des SS-Apparats - im NS-System gemacht wurden. In diesem Zusammenhang ging Trevor-Roper auch nicht auf die Terrorpolitik der SS in Deutschland und nicht ausführlich auf die Massenverbrechen von Himmlers SS-Apparat vor allem an der jüdischen Zivilbevölkerung in den südosteuropäischen Ländern ein.

So schrieb Trevor-Roper unter anderem zwar ausführlich zum Krankheitszustand Himmlers, aber auf die nationalsozialistische Ausrottung der Juden und zu den Konzentrationslagern ging er nur insoweit ein, als es um die Thematisierung der durch die persönliche Beziehung zwischen dem sogenannten Hofarzt Kersten zu Himmler möglich gewordenen Rettungsaktion von Juden am Ende des Zweiten Weltkrieges durch die schwedische Regierung ging. Zwar sprach er hierbei im Kontext der Judenvernichtung von einem »System des Terrors« und mit Blick auf Himmler von einem »Erzverfolger der Juden«. Allerdings dienten diese historischen Fakten nicht in irgend- 
einer Form dazu, ein näheres Bild des >Dritten Reiches` zu zeichnen und analytisch ausgerichtete Aussagen zum NS-System zu machen. Einzelne historische Detailinformationen über Himmler, den SS-Apparat und die »udenpolitik« hatten in der Veröffentlichung im Grunde genommen den gleichen Stellenwert wie einzelne Plaudereien aus den Memoiren von Kersten. Auch bestimmte Aussagen von Kersten wurden von Trevor-Roper kritiklos wiedergegeben: wie zum Beispiel die Behauptung, dass sich Himmler im Laufe der Zeit dem Einfluss seines Arztes nicht entziehen konnte und dieser sogar eine Machtposition einnahm, die es dem Chef der deutschen Polizei nicht mehr möglich machte, Kersten »drohen zu können«.

Letzten Endes drängt sich im Nachhinein der Eindruck auf, dass die Veröffentlichungen von Trevor-Roper im Monat»populäre «Vorstellungen vom zeitgeschichtlichen Gegenstand Nationalsozialismus bediente, die auch und vor allem in der deutschen Bevölkerung grassierten. Substanzielle Erkenntnisse zum NS-Herrschaftssystem enthielten weder Himmlers Leibarzt noch die meisten anderen Veröffentlichungen von ihm.

Im Gegensatz hierzu stand neben dem Aufsatz Hitler und Franco. Warum nahm Spanien nicht am Kriege teil?, der ausführlich auf Hitlers außenpolitische Ziele einging, in allererster Linie der hervorragende Beitrag Martin Bormann von Trevor-Roper. Der Bormann-Aufsatz enthielt sicherlich mit Blick auf die Beschreibung und Analyse des NS-Systems im Monat, neben den genannten Beiträgen von Lüthy, Arendt und von Westphal, die substanziellsten Ausführungen und Einschätzungen. Bemerkenswert an dem Beitrag von Trevor-Roper war vor allem die Tatsache, dass er sich hier von seiner genuin hitlerzentrierten Perspektive auf den »Führerstaat« gelöst hatte und einen nicht ausschließlich personalistischen Forschungsansatz vertrat. Insofern war er imstande, ein facettenreiches, nicht monolithisches Bild vom NS-Herrschaftssystem zu zeichnen. Selbstverständlich stand die Person Martin Bormann sowie dessen politische Karriere im Zentrum der Ausführungen. Allerdings erfolgte die Beschreibung des Aufstiegs von Bormann von einem snormalen 1920er-Jahren zu einem der einflussreichsten NS-Funktionäre in den 1930er-Jahren und besonders in den Jahren des Zweiten Weltkriegs grundsätzlich mit Blick auf einige wichtige Institutionen des NS-Systems. Trevor-Roper zeigte nämlich genau, wie sich Bormann aufgrund verschiedener verwaltungsspezifischer Aufgaben in der NSDAP nicht nur in dieser Institution »bald unentbehrlich « machte, sondern durch seine nach der Machteroberung im Jahre 1933 eingenommenen mehrfachen Funktionen - Reichsleiter der Partei, General der SS und als Stabsleiter beim Stellvertreter des Führers und Leiter der Parteikanzlei, Rudolf Heß - auch und vor allem bei Hitler selbst. Vor diesem Hintergrund zeigte Trevor-Roper in seiner Analyse, worin in erster Linie die institutionellen Voraussetzungen dafür lagen, dass Bormann qua Funktion insbesondere in den Kriegsjahren ein originäres Machtzentrum im Nationalsozialismus darstellte: nämlich durch seine bürokratische Schlüsselstellung in der NSDAP und seine, wie er schrieb, "persönliche Unentbehrlichkeit beim Führer«.

Insbesondere nachdem Bormann 1941 offiziell als Nachfolger von Heß zum Chef der Parteikanzlei und zum persönlichen Sekretär von Hitler ernannt wurde, gelang nach Meinung Trevor-Ropers sein »Aufstieg in die herrschende Oligarchie» des Nationalsozialismus. Denn seine Machtstellung beruhte, wie er eindrucksvoll zeigte, vor allem darauf, dass Bormann Einblick in die täglichen Akteneingänge der verschiedenen Instanzen und Parteidienststellen hatte und somit die Möglichkeit besaß, sich immer dann einzuschalten und in seinem persönlichen Sinne zu intervenieren, wenn 
er es für notwendig erachtete; insbesondere natürlich um seine Position gegenüber anderen NS-Funktionären und NS-Instanzen gegenüber dem »Führer« zu behaupten. Diese Interpretation, der zufolge es sich beim »Hitler-Staat« letzten Endes um ein oligarchisches Regime gehandelt hatte, an dessen Spitze freilich Hitler als unumstrittener »Führer« stand, machte den Beitrag im Folgenden im Hinblick auf die Analyse des Nationalsozialismus im Monat zu einer absoluten Ausnahme: einerseits durch die Beschreibung und Analyse von zentralen Instanzen des NS-Systems, vor allem der »Parteikanzlei«, die ansonsten im Monat im Kontext der Auseinandersetzung mit der »Hitler-Diktatur« nicht berücksichtigt wurden; andererseits durch den Blick auf rivalisierende Institutionen und mithin auf ein Grundprinzip des NS-Herrschaftssystems. Trevor-Roper zeigte ein personell-institutionelles Kompetenzchaos an und konnte den hieraus resultierenden ständigen Konkurrenzkampf führender NS-Machtträger genau zur Sprache bringen. Nicht zuletzt seine Schilderung, wie Bormann insbesondere von Anfang 1941 bis zum Ende des Zweiten Weltkrieges seine Machtposition ständig ausbauen konnte, weil er beim Kampf mit seinen persönlichen Feinden und Rivalen wie Himmler, der Mitte des Jahres 1943 zum Minister des Innern aufstieg und folglich eine für Bormann "gefährliche Macht erlangte« - eine sogenannte Bündnispolitik mit anderen einflussreichen NS-Funktionären einging, die selbst grundsätzliche Gegner« (wie Goebbels) nicht ausschloss, um beim Kampf zur Verteidigung seiner Stellung bei Hitler gemeinsam gegen einen »neuen Feind« (wie Speer) vorzugehen, zeigte nicht nur die (Herrschafts-)Methode von Bormann, sondern auch ein Grundprinzip des »Führerstaates«.

Durch das Fokussieren des Konkurrenzkampfes führender NS-Funktionäre untereinander, also unterhalb der unumstrittenen Stellung des »Führers«, und die Hervorhebung der zentralen Bedeutung von Bormann, der zum »eigentlichen Herrscher über die nächste Umgebung Hitlers« avancierte, so Trevor-Roper, stellte der Beitrag Martin Bormann eine bedeutende Analyse des NS-Systems dar. In diesem Zusammenhang nahm diese Veröffentlichung des NS-Experten in der Zeitschrift Der Monat eine absolute Sonderrolle ein, weil ausnahmsweise der beschreibende und analytische Blick nicht ausschließlich auf Hitler geworfen wurde.

Indes: Wie in seinen anderen Veröffentlichungen zum >Dritten Reich<, in denen Trevor-Roper nicht - oder wie in Himmlers Leibarzt nur am Rande - auf die »udenpolitik« des Regimes einging, spielte für ihn auch der Antisemitismus als wesentliches Element der bormannschen Weltanschauung keine Rolle. Im Zusammenhang mit der Regimekrise anlässlich des Attentats auf Hitler war zwar bei ihm auch von Bormanns fanatischem Hass gegen die Kirchen die Rede, der sich seit Juli 1941 radikalisiert hatte, allerdings bezogen sich seine Ausführungen ausschließlich auf das starke Ressentiment Bormanns (sowie der NSDAP) auf das Christentum. Auch die NS-Massenverbrechen und insbesondere die Vernichtung der europäischen Juden besaßen für TrevorRoper insgesamt keine grundsätzliche Bedeutung bei der Beschreibung und Analyse des NS-Herrschaftssystems.

Den eigentlichen Schwerpunkt der Veröffentlichungen Hofers zum Nationalsozialismus bildete die Rezension von Büchern aus den 1950er-Jahren zu dem Thema Widerstand des 20. Juli. Für die Analyse des Nationalsozialismus im Monat besitzt aber aufgrund der eingenommenen (Forschungs-)Perspektive des Autors sowie der historischen Detailaussagen zur Genesis der NS-Herrschaft der Aufsatz Der »völkische Rechtsstaat«. Zur nationalsozialistischen Rechtspolitik aus dem Jahre 1959 eine größere Bedeu- 
tung. Er setzte sich hierbei allerdings nicht mit den konkreten politisch-historischen Bedingungen der nationalsozialistischen Machteroberung auseinander, die nach seiner Auffassung ausdrücklich als eine »Revolution« anzusehen war.

Bereits zu Beginn seines Beitrages stellte der NS-Experte Hofer fest, dass es ihm um die Analyse eines Herrschaftssystems zu tun war, das extrem auf der "persönlichen Diktatur eines Mannes« aufgebaut war. Vor diesem Hintergrund ging es ihm zwingend um die Untersuchung der »Anschauungen dieses entscheidenden Mannes«. Diese einleitenden Sätze zeigten Hofer als einen Vertreter der Zeitgeschichte, der bei der Erforschung bzw. der Untersuchung der Geschichte des Nationalsozialismus ausdrücklich einen genuin hitlerzentrierten Ansatz vertrat. Der extrem auf Hitler bezogene Blick auf das NS-System korrespondierte mit einer totalitarismustheoretischen Perspektive, die darin zum Ausdruck kam, dass Hofer neben seiner Vorstellung der »unumschränkten Diktatur« Hitlers, vom »Staate Hitlers« oder vom »totalen Führerstaat" im Großen und Ganzen den analytischen Schwerpunkt auf die sogenannte kleine kriminelle Führung legte: also neben Hitler auf Göring, Himmler und Heydrich sowie - ganz im Sinne der populären klassischen Totalitarismustheorien - auf die SS und die Gestapo sowie der NSDAP als einer »herrschenden Staatspartei«; zudem wurde die Justiz als eine Institution beschrieben, die letzten Endes vor dem Hintergrund der »nationalsozialistischen Rechts- und Staatsauffassung« und dem »Willen des Führers« ausschließlich als ein »Organ des Terrors« charakterisiert wurde. Da es sich nach Auffassung von Hofer bei der Machteroberung des Nationalsozialismus um eine Revolution gehandelt hatte, beschrieb er in diesem Kontext die Jahre der Machtkonsolidierung des Regimes und den Aufstieg der politischen Polizei unter der Führung von Himmler auch nicht als einen historisch-politisch offenen Prozess. Mithin ging Hofer nicht darauf ein, dass Hitler am 30. Januar 1933 vom Reichspräsidenten Hindenburg zum Reichskanzler ernannt worden war und es sich infolgedessen um eine »legale Machtergreifung " gehandelt hatte. Die Rede war somit nicht davon, dass Hitler nicht aus eigener Stärke an die Schaltstelle der Macht kam, sondern durch führende Exponenten der bürgerlich-konservativen Elite, die ihn im Rahmen ihres "Zähmungskonzeptes« als ein Instrument in ihrem Kampf gegen die Weimarer Republik sowie die linken Parteien benutzen wollten, an dessen Ende die Errichtung eines dauerhaften autoritären Präsidialregimes stehen sollte.

Vor diesem Hintergrund wird im Nachhinein verständlich, dass Hofer im Kontext seines mit einer totalitarismustheoretischen Perspektive auf die »Hitlerdiktatur " geschriebenen Beitrages eine eindeutige, planmäßige, extrem zielgerichtete (Terror-) Politik Hitlers in den ersten Jahren nach der »Machtergreifung« hin zu einem nach völkischen Rechtsprinzipien aufgebauten »Führerstaat« suggerierte, die die tatsächliche innenpolitische Entwicklung in Deutschland nach dem 30. Januar 1933 außer Acht ließ. So konnte nach der Lektüre des Aufsatzes unter anderem der Eindruck entstehen:

1. Dass Hitler nach dem 30. Januar 1933 sozusagen frei schalten und walten konnte und mithin eine autonome genuin nationalsozialistische Politik betreiben konnte. Somit kam bei Hofer nicht zur Sprache, dass der neuernannte Reichskanzler Rücksicht nehmen musste auf die spezifischen Bedingungen des demokratischen Systems von Weimar und insbesondere auf die bürgerlich-konservativen Kräfte im neuen Präsidialkabinett. Die NSDAP war hier nämlich nur durch zwei Mitglieder vertreten: den Reichsinnenminister Wilhelm Frick und Hermann Göring, der 
als Minister ohne Geschäftsbereich unter anderem die Aufgabe des kommissarischen preußischen Innenministers übernahm. Mithin wurde von Hofer auch die zentrale Bedeutung ebendieser Besetzung zweier Schlüsselposten auf dem Weg zum anvisierten »Führerstaat « nicht in den Blick genommen, denn damit sollte der Zugriff auf die polizeilichen Sicherheitsapparate gesichert werden, und zwar insbesondere in der Reichshauptstadt sowie in Preußen. Hiermit sollten die Weichen gestellt werden für das zentrale Ziel: eine einheitliche politische Reichspolizei des späteren NS-Regimes zu errichten.

2. Dass die aus Anlass des Reichstagsbrandes verabschiedete Verordnung "Zum Schutze von Volk und Staat« vom 28. Februar 1933, die, wie Hofer richtig feststellte, die bürgerlichen Grundrechte außer Kraft setzte und den »Ausnahmezustand« ins Leben rief, ausschließlich auf dem »Willen des Führers« beruhte. Mithin wurde nicht ausdrücklich angesprochen, dass die von Hitler vorgeschlagene Notverordnung vom Regierungskabinett unter Berufung auf den Artikels 48 der Weimarer Verfassung verabschiedet und durch die Unterschrift vom Reichspräsidenten Hindenburg abgesegnet wurde. Zudem ging Hofer in diesem Kontext nicht auf die Bedeutung des vom Deutschen Reichstag am 23. März 1933 beschlossenen »Ermächtigungsgesetztes« ein, mit dem Hitler »absolute Handlungsfreiheit« bekam.

3. Dass die Entwicklung der staatlichen politischen Polizei unter der Führung von Himmler nach der sogenannten nationalsozialistischen Revolution im Grunde genommen den Charakter einer "Einbahnstraße« aufwies, d.h., keinen offenen Prozesscharakter besaß. Infolgedessen wurde nicht beleuchtet, dass die gesamte Entwicklung der Polizei, besonders der politischen Polizei, in Deutschland nach der Reichstagswahl vom 5. März 1933 durch die Tatsache, dass die Regierungskoalition - bestehend aus der NSDAP und der DNVP - nicht in allen deutschen Ländern die parlamentarische Mehrheit erreichte, keineswegs eindimensional und zwangsläufig verlief. So kam von Hofer beispielsweise auch nicht zur Sprache, dass Himmlers Aufstieg zum Chef der deutschen Polizei nicht von Anfang eindeutig entschieden war. In diesem Zusammenhang wurde einerseits im Hinblick auf den Zugriff auf die politische Polizei die spezifische institutionelle Rivalität zwischen dem Reichsminister des Inneren und der SS sowie zwischen der SS und der SA nach der »Machtergreifung « nicht genau angesprochen. Andererseits auch nicht auf die besondere Rivalität zwischen Himmler und Göring insistiert, der nämlich nach dem 30. Januar die politische Polizei in Preußen zu einem Machtinstrument ausgebaut hatte und infolge einer komplexen innenpolitischen bzw. machtpolitischen Entwicklung bereits im Frühjahr 1934 Himmler - dem bis dahin die politische Polizei in Bayern unterstellt war - als seinen Stellvertreter akzeptieren musste und nur noch das "formelle Kommando« (Hans Buchheim) besaß. In diesem Kontext stellte die Ernennung von Heydrich zum Chef der Gestapo zweifelsohne eine wesentliche Bedingung für den Aufstieg Himmlers dar.

4. Dass im Zusammenhang mit der »nationalsozialistischen Rechtspolitik« die »Judenpolitik« des Nationalsozialismus nach der »Machtergreifung« keine überragende Bedeutung besaß. Denn auf die konkreten antisemitischen Maßnahmen des sogenannten kriminellen Staates in Deutschland im Laufe der 1930er-Jahre kam Hofer nur im Vorbeigehen zu sprechen. Von der nach dem Überfall auf die Sowjetunion in Gang gekommenen »Endlösung der Judenfrage« (bei Hofer unter die allgemeine Kategorie »Massenverbrechen« rubriziert) sowie dem System der Kon- 
zentrations- und Vernichtungslager im Osten war bei ihm auch nicht ausdrücklich die Rede.

Gleichwohl besitzt der Aufsatz Der »völkische Rechtsstaat « für die hier interessierende Analyse des Nationalsozialismus im Monat eine hohe Bedeutung, und zwar durch die Tatsache, dass ausdrücklich die konkrete innenpolitische Entwicklung respektive Situation in Deutschland in den 1930er-Jahren beleuchtet wurde, sowie durch den Versuch, zu erklären, wie die nationalsozialistische Weltanschauung, d. h. in erster Linie Hitlers »Rechtsvorstellung«, in politische (Rechts-)Praxis umgesetzt wurde. Zudem enthielt Hofers Aufsatz aus dem Jahre 1959 eine Fülle von konkreten Detailinformationen und -aspekten zur Geschichte des >Dritten Reiches` nach der Machteroberung so etwa zur Bedeutung von Intellektuellen bzw. von Juristen wie Carl Schmitt für den "Führerstaat« -, die man in den Jahren zuvor im Monat als Leser dieser Zeitschrift letzten Endes nur »nebenbei« erfuhr. So war zum Beispiel seine Auffassung über die politische Bedeutung der sogenannten Reichstagsbrandverordnung, der Verordnung zum Schutz von Volk und Staat vom 28. Februar 1933, für das In-Gang-Kommen der Terrorpolitik wichtig. Mit dieser Verordnung, die quasi die "Verfassungsurkunde« (Ernst Fraenkel) des >Dritten Reiches darstellte und die bis zum Ende der NS-Herrschaft galt, wurde nämlich tatsächlich die »Rechtsgrundlage« geschaffen, auf der dann, wie Hofer richtig feststellte, die politische Polizei zur »Abwehr kommunistischer staatsgefährdender Gewaltakte« ihre »Maßnahmen« auf andere "Gegner» des Regimes ausgedehnte. Für Hofer diente die gleiche Verordnung auch zum Kampf gegen die »religiösen Sekten«, sprich den sogenannten Bibelforscher und gegen die evangelische und katholische Kirche sowie letztlich gegen alle Personen, die unter anderem der Zusammenarbeit mit den Kommunisten verdächtig wurden, sodass letztlich »jeder« als »potentieller Staatsfeind« betrachtet werden konnte In diesem Zusammenhang skizzierte er bemerkenswerterweise auch, wie die »Willkür der politischen Polizei« auch dazu führte, dass auf der »rechtlichen Grundlage« für die sogenannte Schutzhaft auch die »unbefristete Einlieferung in ein Konzentrationslager« möglich war, die seiner Auffassung dem Ziel des NS-Regimes dienten, die »Feinde der politischen Ordnung aus der Volksgemeinschaft auszuschließen«.

Kurzum: Hofers Veröffentlichung stellte im Zusammenhang mit der Analyse des Nationalsozialismus im Monat insofern eine Ausnahme dar, als der NS-Experte ausdrücklich auf einzelne Aspekte und Details der nationalsozialistischen Innenpolitik nach der Machteroberung einging. Hierbei lag der eigentliche Schwerpunkt auf einer Analyse der politischen Polizei bzw. der SS und der Gestapo unter der Führung von Himmler in den 1930er-Jahren. Die konkrete nationalsozialistische »Judenpolitik“ spielte allerdings in seinem Beitrag keine Rolle. Analog zu seinem in Deutschland überaus populären, in einer hohen Auflage erschienenen Buch Der Nationalsozialismus. Dokumente 1933-1945 $5^{336}$ dominierte ein hitlerzentrierter Forschungsansatz, der mit einer totalitarismustheoretischen Perspektive korrespondierte. 